\title{
Surface Reactivity and Quantum-Size effects on the Electronic Density Decay Length
} of ultrathin Metal Films

\author{
N. Binggeli ${ }^{1,2}$ and M. Altarelli ${ }^{1,3}$ \\ 1 The Abdus Salam International Center for Theoretical Physics, Trieste 34014, Italy \\ ${ }^{2}$ INFM-CNR DEMOCRITOS National Simulation Center, Trieste 34014 , Italy \\ 3 European XFEL Project Team, DESY, Notkestraße 85, 22607, Hamburg, Germany
}

\begin{abstract}
The origin of the correlation between surface reactivity and quantum-size effects, observed in recent experiments on the oxidation of ultrathin magnesium films, is addressed by means of ab initio calculations and model predictions. We show that the decay length in vacuum of the electronic local density of states at the Fermi energy exhibits systematic oscillations with film thickness, with local maxima induced when a quantum well state at $k_{\|}=0$ crosses the Fermi energy. The predicted changes in the decay length are expected to have a major impact on the electron transfer rate by tunneling, which has been proposed to control the initial sticking of $\mathrm{O}_{2}$ in the oxidation process.
\end{abstract}

PACS numbers: 73.20.At, 73.21.Fg, 82.65.+r, 68.43.-h

There is considerable interest in identifying methods to tailor the chemical reactivity of surfaces, a crucial factor in many technologically relevant surface phenomena, including oxidation and catalysis. The recent observation of a correlation between quantum-size effects and the surface reactivity of ultrathin metal films [1] is an exciting development in this area, both because of its fundamental interest for a quantitative understanding of the structure-size dependence of the reactivity and in view of the importance of nano-scale and low-dimensional structures in modern technology. Experimentally, oscillations were observed in the oxidation rate of ultrathin $\mathrm{Mg}(0001)$ films on $\mathrm{W}(110)$, as a function of film thickness, with the largest oxidation rate occurring when a quantum-well state was found to cross the Fermi energy, in photoemission $(\mathrm{PE})$ spectra [1] taken near normal incidence. In particular, the changes observed in the initial oxidation rate - when most of the film was still metallic, were found to be dramatic. The origin of these changes has not been established yet. The changes in reactivity were suggested to be due to a change in the density of states (DOS) at, or near the Fermi energy $E_{F}[1]$, as the oscillations in the reactivity were found to correlate with the oscillations in the PE intensity at $E_{F}$. However, unlike the normal-incidence PE intensity, which measures the partial DOS at $k_{\|}=0$, the total DOS of the films at $E_{F}$ is bound to exhibit a monotonic, staircase-like increase with film thickness [2], and cannot thus simply account for the oscillations in the reactivity.

Here we propose a theoretical interpretation of these observations, in which the decay length in the vacuum of the electronic local density of states around the Fermi energy is identified as the key parameter responsible for the changes in the reactivity. On the basis of ab initio calculations, inspired by model predictions for quantum well states, we show that the decay length, $\lambda$, of the local density of states at the Fermi energy exhibits an oscillatory behavior with film thickness, with local maxima present when the highest occupied quantum-well state at $k_{\|}=0$ is closest to the Fermi energy. The predicted changes in the decay length are significant. They are expected to have a direct, exponential impact on the electron transfer rate by tunneling, which has been proposed to control the initial sticking of $\mathrm{O}_{2}$ on the metal surface [3]. The changes in $\lambda$ should be observable by scanning tunneling microscopy (STM), near terrace steps, on surfaces characterized by regions of different film thicknesses [1].

Model predictions for $\lambda$.-Within an independent electron description and considering, for simplicity, a squarewell potential along the $z$ direction, normal to the thin film, with a constant depth $V$ and a variable width $L$ (film thickness), the electronic states are solutions of a separable problem in the $z$ and $(x, y)$ variables, and read:

$$
\psi_{n, k_{x}, k_{y}}^{E}(x, y, z) \sim \chi_{n}(z) \cdot e^{i\left(k_{x} \cdot x+k_{y} \cdot y\right)},
$$

with energy $E=E_{n}+\hbar^{2}\left(k_{x}^{2}+k_{y}^{2}\right) / 2 m^{*}$, where $m^{*}$ stands for the electron effective mass. $E_{n}$ and $\chi_{n}(z)$ are the eigenvalues and eigenstates of the one-dimensional square-well potential problem, with the following properties:

$$
\chi_{n}(z) \sim e^{-\alpha_{n} \cdot z}
$$

for $z \geq L / 2$, assuming the $z$ origin at the center of the film, and:

$$
\alpha_{n}=\left(\sqrt{2 m^{*}} / \hbar\right) \cdot \sqrt{-E_{n}},
$$

where the zero of energy is taken at the vacuum level. In the three-dimensional problem, $E_{n}$ coincides with the energy $E$ of the subband state $n$ at $k_{\|}=0$, measured relative to the vacuum level.

All states $\psi_{n, k_{x}, k_{y}}^{E}$ belonging to subband $n$, with energy $E \geq E_{n}$, are thus characterized by the same decay length $1 / \alpha_{n} \sim 1 / \sqrt{-E_{n}}$, for a given film thickness $L$. If $E_{F}$ is located between the levels $E_{n}$ and $E_{n+1}$, the dominant decay length of the electronic states at $E_{F}$ is $1 / \alpha_{n}$, namely the decay length of the highest occupied band 
state at $k_{\|}=0$. With increasing width $L$ of the well, the energies of the quantum-well states decrease with respect to $E_{F}$; hence, the decay length $\lambda$ of the electronic density at $E_{F}$ first decreases as $1 / 2 \alpha_{n} \sim 1 / \sqrt{-E_{n}}$, until the next quantum-well state at $\bar{\Gamma}$ crosses $E_{F}$, at which point $\lambda$ increases to the new value $1 / 2 \alpha_{n+1} \sim 1 / \sqrt{-E_{n+1}}$. The decay length then decreases again with increasing $L$, displaying systematic oscillations with $L$. Considering next a discrete number of atomic layers, the periodicity of the crossing of the Fermi energy may be derived from the Bohr-Sommerfeld rule, which for $\mathrm{Mg}(0001)$ yields a periodicity of 7.7 monolayers (ML) [4].

From the model description, we expect thus oscillations in $\lambda$, with local maxima occurring when the highest occupied quantum-well states at $\bar{\Gamma}$ is closest to $E_{F}$. A more realistic description of the system, however, is clearly needed to confirm the robustness of this behavior, and to quantify the effect. In particular, electron interaction, atomic orbitals and bonds, surface states, and the presence of the substrate, are all factors which are expected to influence the decay length $\lambda$. We have therefore examined the effect of quantum confinement on $\lambda$ by means of $a b$ initio calculations performed for epitaxial $\mathrm{Mg}(0001)$ films on W(110) and also, for comparison, for the corresponding unsupported $\mathrm{Mg}(0001)$ films in vacuum.

First-principles results.-The calculations were performed within density functional theory, using the Perdew-Burke-Ernzerhof exchange-correlation functional [5], Troullier-Martins pseudopotentials [6], and a planewave basis set. The $\mathrm{Mg}$ pseudopotential was generated in the atomic configuration: $3 s^{1.8} 3 p^{0} 3 d^{0.2}$, using as coreradii cutoff: $r_{\mathrm{s}, \mathrm{p}, \mathrm{d}}=2.8$ a.u.. For $\mathrm{W}$ we treated the $6 s$, $6 p$, and $5 d$ orbitals as valence states, using the same parameters as in Ref. [7]. The overlap between valence and core electrons was accounted for using the non-linear-core correction to the exchange-correlation potential [8]. A kinetic-energy cutoff of 49 Ry was used for the plane-wave expansion of the electronic orbitals of the $\mathrm{Mg} / \mathrm{W}$ systems. For the isolated $\mathrm{Mg}(0001)$ slabs we used a kinetic-energy cutoff of 14 Ry. The Brillouin-zone sampling was done with a Monkhorst-Pack (MP) grid [9], and we used a Gaussian smearing of the electronic levels of $0.02 \mathrm{Ry}$ to determine the Fermi energy. The films were modeled using slab geometries in supercells. For the $\mathrm{Mg}$ films on $\mathrm{W}$, we considered slabs containing $7 \mathrm{~W}(110) \mathrm{ML}$, terminated by 5 to $12 \mathrm{Mg}(0001) \mathrm{ML}$ on one side, and by $2 \mathrm{Mg}$ (0001) ML on the other side. The Mg bilayer was introduced in order to avoid the presence of an electric field-due to the different work functions of $\mathrm{W}$ and $\mathrm{Mg}$ - in the vacuum regions separating the periodic images of the slab. We used vacuum regions with a minimal thickness of 20.1 $\AA$, in order to obtain well-converged values of $\lambda$.

Experimentally, above $3 \mathrm{ML}$, the $\mathrm{Mg}$ films are known to grow epitaxially on W(110) [4], with lattice parameters corresponding to their bulk values [10]. The mismatches between the experimental in-plane lattice parameters of
$\mathrm{Mg}(0001)\left(a_{\mathrm{Mg}}=3.21 \AA, b_{\mathrm{Mg}}=\sqrt{3} a_{\mathrm{Mg}}\right)$ and $\mathrm{W}(110)$ $\left(a_{\mathrm{W}}=3.16 \AA, b_{\mathrm{W}}=\sqrt{2} a_{\mathrm{W}}\right)$ are $1.6 \%$ and $\sim 20 \%$ [4]. To model such epitaxial systems, a commensurate interface atomic structure is needed in the calculations. The inclusion of dislocations would require prohibitively large lateral dimensions of the supercell, and the details of the atomic structure at the interface are unknown in any case. In order to simulate an unstrained $\mathrm{Mg}$ film on $\mathrm{W}(110)$, we thus elected to laterally strain the W(110) slab to the in-plane lattice parameters of $\mathrm{Mg}(0001)$. The epitaxial alignment was made by positioning the atoms of the first W(110) layer, adjacent to the $\mathrm{Mg}$, in the continuation of the $\mathrm{Mg}$ (0001) hcp lattice. The three outermost layers of the $\mathrm{Mg}$ film were relaxed, while the other interlayer spacings were kept frozen at their bulk position (using the theoretical values of the bulk lattice constants: $a_{\mathrm{Mg}}=3.19 \AA, c_{\mathrm{Mg}}=5.18 \AA$, and $a_{\mathrm{W}}=3.21 \AA$ ). It should be noted, however, that the $\operatorname{Mg}(0001)$ surface relaxations are small $[11,12]$, and were found to have a negligible impact on $\lambda$. The self-consistent calculations were carried out using a $(20,20,1)$ MP grid. For the local density of states, we used a $(48,48,1)$ grid centered at $\Gamma$, with a Gaussian level smearing of 0.005 Ry. The decay length was derived from a fit, assuming an exponential decay of the local density of states at distances beyond $\sim 2.15 \AA$ from the outermost atomic plane.

In Fig. 1, we report the calculated decay length $\lambda$ of the $\mathrm{Mg}$ films, as a function of film thickness. The results are shown for both the $\mathrm{Mg}$ films on $\mathrm{W}$ and the isolated $\mathrm{Mg}$ films in vacuum. The behaviors are very similar in the two cases. The decay length exhibits a pronounced oscillation, with a maximum at 9 layers and a minimum at 6-7 layers. The presence of the tungsten substrate tends to reduce the amplitude of the variation of $\lambda$, from $17 \%$ to $10 \%$, but has no significant impact on the position of the extrema. The densities of states at $\bar{\Gamma}$ of the $\mathrm{Mg}(0001)$ films on W(110) are displayed in Fig. 2. With increasing film thickness, an unoccupied quantum-well state crosses the Fermi energy at $\sim 9$ layers. This coincides with the maximal decay length $\lambda$ found in Fig. 1, consistent with the model prediction. In Fig. 2, we also reported the energy positions of the electronic states at $\bar{\Gamma}$ obtained from the calculations for the isolated $\mathrm{Mg}$ slabs in vacuum. Also in this case, a quantum-well state is found to pass through the Fermi energy at $\sim 9$ layers, which corresponds to the largest $\lambda$ obtained in Fig. 1 . We note that we have also examined the variation of the workfunction of the $\mathrm{Mg}$ films on $\mathrm{W}$ with the number of $\mathrm{Mg}$ layers [13], as quantum size effects can be expected on this value [14]. For the $\mathrm{Mg}$ films on $\mathrm{W}$, however, the calculated changes in the work function are found to be very small, namely a variation of $0.05 \mathrm{eV}$ in the range 5-12 layers. With increasing thickness, the workfunction first decreases, in the range 5-8 layers, from 3.74 to 3.69 $\mathrm{eV}$, and then increases, in the range 8-10 layers, from 3.69 to $3.72 \mathrm{eV}$, and then saturates at $3.72 \mathrm{eV}$ for higher 
ergy range $\left[E_{F}-4 \mathrm{eV}, E_{F}\right]$, in Fig. 2, compare well with the near-normal-incidence PE measurements [1], except for a systematic shift, by about $+2 \mathrm{Mg} \mathrm{ML}$, of the theoretical spectra with respect to the measured spectra. In our calculations, the first quantum-well state with energy higher than the surface/interface states SS, enters the occupied-state spectrum at a $\mathrm{Mg}$ thickness of 8-9 ML. In the experiment, this state is found, instead, to enter the valence-band spectrum at a nominal thickness of 6-7 ML. The same shift is observed between the calculated maximum/minima of $\lambda$ and the experimental maximum/minima of the reactivity. Such a shift is probably due to the use of a commensurate, dislocation-free interface atomic structure, in the calculations, to describe a heavily lattice-mismatched epitaxial system. The presence of strained $\mathrm{Mg}$ layers or dislocations at the interface could result in the entrance of a quantum-well state at a smaller value of the number of layers of the $\mathrm{Mg}$ film.

The changes in $\lambda$ we predict should be observable, e.g., by STM near terrace steps on a Mg surface characterized by regions of different film thicknesses [1]. Also, He scattering experiments have indicated an apparent stepheight oscillation in the layer-by-layer growth of $\mathrm{Pb}$ on Ge due to quantum-size effects [18]. Such changes were recently shown to be due mostly to a displacement of the topmost layer charge density [19], in contrast to previous interpretations in terms of a displacement of the surface atomic planes. This recent analysis corroborates thus the present finding on the behavior of the decay length of the $\mathrm{Mg}$ films. We note that the changes in the decay length of the density of states near the Fermi energy may also be related to a recent observation of quantum-size effects on the chemisorption properties of $\mathrm{Cu}(001)$ thin films [20]. In addition, layer-KKR (Korringa-Kohn-Rostoker) calculations [21] have indicated that the lifetime of negative ionic states of molecules, adsorbed on supported metal thin films varies with the thickness of the film, through coupling to quantum-well states. This effect (not yet measured, to our knowledge) was associated, in the calculations, with oscillations in the amplitude, at the position of the molecule, of the density of empty states above $E_{F}$. This calculated behavior, consistent with the trend we find for $\lambda$, also supports the interpretation we propose for the reactivity changes.

In conclusion, on the basis of ab initio calculations for $\mathrm{Mg}(0001)$ ultrathin films on $\mathrm{W}(110)$, we have shown that the decay length in vacuum of the electronic local density of states at the Fermi energy exhibits substantial oscillations with the film thickness. The decay length is maximal, as a function of film thickness, when a quantum-well state passes through the Fermi energy. The changes we predict in the decay length are expected to have a major impact on the tunneling rate in the electron transfer mechanism, which is believed to control the initial sticking of $\mathrm{O}_{2}$ on the $\mathrm{Mg}$ surface. We therefore propose that the experimental tuning of the reactivity is due to quan- tum oscillations in the electronic density decay length, which should be observable by STM.

We thank L. Aballe, A. Barinov, M. Kiskinova, N. Stojic, and G. Trimarchi for helpful discussions. The computations have been performed using the PWscf package [22]. We acknowledge support by the INFM within the framework of the "Iniziativa Trasversale Calcolo Parallelo".

[1] L. Aballe, A. Barinov, A. Locatelli, S. Heun, and M. Kiskinova, Phys. Rev. Lett. 93, 196103 (2004).

[2] See, e.g., J. C. Boettger and S. B. Trickey, Phys. Rev. B 45, 1363 (1992); the increase of the film DOS is implicit when the DOS is normalized to the number of atoms in the film.

[3] A. Hellman, B. Razaznejad, Y. Yourdshahyan, H. Ternow, I. Zoric, B. I. Lundqvist, Surface Science 532-535, 126 (2003).

[4] F. Schiller, M. Heber, V. D. P. Servedio, and C. Laubschat, Phys. Rev. B 70, 125106 (2004); and references therein.

[5] J. P. Perdew, K. Burke, and M. Ernzerhof, Phys. Rev. Lett. 77, 3865 (1996).

[6] N. Troullier and J. L. Martins, Phys. Rev. B, 43, 1993 (1991).

[7] K. W. Kwak, M. Y. Chou, and N. Troullier, Phys. Rev. B 53, 13734 (1996).

[8] S. G. Louie, S. Froyen, and M. L. Cohen, Phys. Rev. B 26, 1738 (1982).

[9] H. J. Monkhorst and J. P. Pack, Phys. Rev. B 13, 5188 (1976).

[10] Based on LEED measurements, L. Aballe, private communication.

[11] P. Staikov and T. Rahman, Phys. Rev. B 60, 15613 (1999); and references therein.

[12] For 5 to $12 \mathrm{Mg} \mathrm{ML}$ on $\mathrm{W}$, the relaxation of the topmost interlayer spacing (the largest relaxation) was found to be in the range +1.3 to $+1.5 \%$.

[13] We employed symmetric slabs with less vacuum, using the electrostatic potential as a reference in the vacuum.

[14] See, e.g., J. J. Paggel, C. M. Wei, M. Y. Chou, D. A. Luth, T. Miller, and T. C. Chiang, Phys. Rev. B 66, 233403 (2002); and references therein.

[15] H. B. Michaelson, J. Appl. Phys. 48, 4729 (1977).

[16] C. M. Wei and M. Y. Chou, Phys. Rev. B 68, 125406 (2003).

[17] C. Bungaro, C. Noguera, P. Ballone, and W. Kress, Phys. Rev. Lett. 79, 4433 (1997).

[18] A. Crottini, D. Cvetko, L. Floreano, A. Morgante, and F. Tommasini, Phys. Rev. Lett. 79, 1527 (1997).

[19] L. Floreano, D. Cvetko, F. Bruno, G. Bavdek, A. Cossaro, R. Gotter, A. Verdini, and A. Morgante, Prog. Surf. Sci. 72, 135 (2003).

[20] A. G. Danese, F. G. Curtis, and R. A. Bartynski, Phys. Rev. B 70, 165420 (2004).

[21] P. J. Rous, Phys. Rev. Lett. 83, 5086 (1999).

[22] S. Baroni, A. dal Corso, S. de Gironcoli, and P. Giannozzi, URL http://www.pwscf.org/. 